Springer Series in Design and Innovation 5

ᄃ

Luis Agustín-Hernándéz

Aurelio Vallespín Muniesa

Angélica Férnáñdez-Morales Editors.
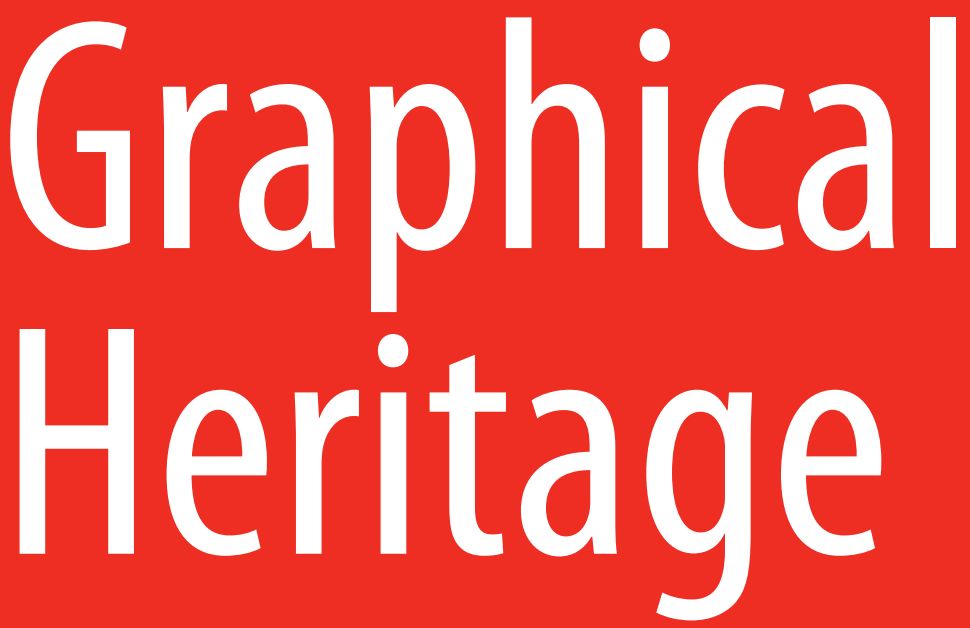

Volume 1 - History and Heritage

Springer 


\section{Springer Series in Design and Innovation}

\section{Volume 5}

\section{Editor-in-Chief}

Francesca Tosi, University of Florence, Florence, Italy

\section{Series Editors}

Claudio Germak, Politecnico di Torino, Turin, Italy

Francesco Zurlo, Politecnico di Milano, Milan, Italy

Zhi Jinyi, Southwest Jiaotong University, Chengdu, China

Marilaine Pozzatti Amadori, Universidade Federal de Santa Maria,

Santa Maria, Rio Grande do Sul, Brazil

Maurizio Caon, University of Applied Sciences and Arts, Fribourg, Switzerland 
Springer Series in Design and Innovation (SSDI) publishes books on innovation and the latest developments in the fields of Product Design, Interior Design and Communication Design, with particular emphasis on technological and formal innovation, and on the application of digital technologies and new materials. The series explores all aspects of design, e.g. Human-Centered Design/User Experience, Service Design, and Design Thinking, which provide transversal and innovative approaches oriented on the involvement of people throughout the design development process. In addition, it covers emerging areas of research that may represent essential opportunities for economic and social development.

In fields ranging from the humanities to engineering and architecture, design is increasingly being recognized as a key means of bringing ideas to the market by transforming them into user-friendly and appealing products or services. Moreover, it provides a variety of methodologies, tools and techniques that can be used at different stages of the innovation process to enhance the value of new products and services.

The series' scope includes monographs, professional books, advanced textbooks, selected contributions from specialized conferences and workshops, and outstanding Ph.D. theses.

Keywords: Product and System Innovation; Product design; Interior design; Communication Design; Human-Centered Design/User Experience; Service Design; Design Thinking; Digital Innovation; Innovation of Materials.

\section{How to submit proposals}

Proposals must include: title, keywords, presentation ( $\max 10,000$ characters), table of contents, chapter abstracts, editors'/authors' CV.

In case of proceedings, chairmen/editors are requested to submit the link to conference website (incl. relevant information such as committee members, topics, key dates, keynote speakers, information about the reviewing process, etc.), and approx. number of papers.

Proposals must be sent to: series editor Prof. Francesca Tosi (francesca.tosi@ unifi.it) and/or publishing editor Mr. Pierpaolo Riva (pierpaolo.riva@ springer.com).

More information about this series at http://www.springer.com/series/16270 


\section{Editors}

Luis Agustín-Hernández

Escuela de Ingeniería y Arquitectura

Zaragoza, Spain

\author{
Angélica Fernández-Morales \\ Escuela de Ingeniería y Arquitectura \\ Zaragoza, Spain
}

\author{
Aurelio Vallespín Muniesa \\ Escuela de Ingeniería y Arquitectura \\ Zaragoza, Spain
}

ISSN 2661-8184

ISSN 2661-8192 (electronic)

Springer Series in Design and Innovation

ISBN 978-3-030-47978-7

https://doi.org/10.1007/978-3-030-47979-4

ISBN 978-3-030-47979-4 (eBook)

(C) The Editor(s) (if applicable) and The Author(s), under exclusive license

to Springer Nature Switzerland AG 2020

This work is subject to copyright. All rights are solely and exclusively licensed by the Publisher, whether the whole or part of the material is concerned, specifically the rights of translation, reprinting, reuse of illustrations, recitation, broadcasting, reproduction on microfilms or in any other physical way, and transmission or information storage and retrieval, electronic adaptation, computer software, or by similar or dissimilar methodology now known or hereafter developed.

The use of general descriptive names, registered names, trademarks, service marks, etc. in this publication does not imply, even in the absence of a specific statement, that such names are exempt from the relevant protective laws and regulations and therefore free for general use.

The publisher, the authors and the editors are safe to assume that the advice and information in this book are believed to be true and accurate at the date of publication. Neither the publisher nor the authors or the editors give a warranty, express or implied, with respect to the material contained herein or for any errors or omissions that may have been made. The publisher remains neutral with regard to jurisdictional claims in published maps and institutional affiliations.

This Springer imprint is published by the registered company Springer Nature Switzerland AG

The registered company address is: Gewerbestrasse 11, 6330 Cham, Switzerland 
Luis Agustín-Hernández ·

Aurelio Vallespín Muniesa .

Angélica Fernández-Morales

Editors

Graphical Heritage

Volume 1 - History and Heritage

黛 Springer 


\section{Preface}

This book, titled 'Graphical Heritage,' contains the contributions to the XVIII International Congress of Architectural Graphic Expression, held at the School of Engineering and Architecture of the University of Zaragoza. The call for papers was answered by 239 contributions, which underwent a blind peer-review process. The initial analysis of the extended abstracts and, subsequently, of the full contributions discarded 59 papers and finally accepted 180 . As a complement to the review performed by international experts, the contributions have been submitted to an anti-plagiarism software check and an examination of their English language academic style, in order to obtain a publication of the highest scientific level.

The geographical origin of the accepted contributions is very varied. With 126 from Spain and 54 from an international origin, it can be said that it is a clear exposition of the most contemporary knowledge in the Architectural Graphic Expression field of research.

As previous congresses, the main theme has been established. This theme has been heritage, understood as architectural heritage, as graphic heritage and as the graphics of heritage; its study, documentation, intervention, conservation, inventory and recovery ultimately point out its value. In support of this theme, invited conferences will be held, including speakers such as Rafael Aranda of RCR Arquitectes, Antonio Almagro, Professor Emeritus of the CSIC or Asunción Hernández, professor at the University of Zaragoza, as well as Luis Franco, architect, Ricardo Usón, PhD architect and Javier Ibargüen, architect, experts in restoration and intervention in architectural heritage.

This congress not only addresses the matter of heritage but every scientific research line related to the area of knowledge, geometry, architectural drawing, color, formal research, representation, diagrams, conceptualization, modeling, rendering, BIM, GIS, cartography, landscape, virtual reality, augmented reality, without forgetting teaching research. 
To collect all these initiatives, the following topics have been considered:

1. Heritage and history.

2. Representation and analysis.

3. Concept and creation.

4. Mapping, cartography and landscape.

5. Teaching innovation.

From drawing, the creator's first decision in architecture, painting or sculpture, as the ability to transfer an idea onto a support, the ability to direct the hand in a specific line, as the first creative element, to virtual reality, the virtual building, far beyond reality. It all is reflected in the communications included in this book and in this congress.

The chapters included in this book, the first in a set of three volumes, pertain to the topic: Heritage and History.

\section{Heritage and History}

The architectural heritage, conceived not only as a series of real estate but as the ensemble of structures that form the physical context in which society has developed throughout history, has been one of the main fields of study of the area of architectural graphic expression throughout the years. Based on this fact, multiple approaches to architectural reality have been possible, whether it was built or not.

Heritage documentation and graphic restitution remain the fundamental basis for its study, protection and recovery, collaborating in this task several systems and tools, from the most traditional ones, such as sketches or stratigraphic studies, to those which use the most up-to-date technical possibilities, such as laser scanning or colorimetric analysis, creating interactions and synergies between tradition and innovation.

The historical studies, archives and graphic representations from the past that have lingered on, as well as the current surveys and constructive research, allow for the realization of virtual reconstructions of missing architectures, even of those that were not built, and reinforce the role of the architecture as a chronicler of the time that generated it.

The virtual graphic representation, with applications such as augmented reality, is a powerful tool both to facilitate research and to disseminate it, allowing for direct reading and interpretation by the observer of the different phases, present, past and future, of the architectural object. 
This topic includes the following:

- Documentation and graphic restitution

- Low tech/high tech: interactions between tradition and innovation

- Techniques to analyze, rebuild and show what no longer exists, or never did

- Virtual and augmented reality to understand and communicate the present, past and future

Luis Agustín-Hernández Aurelio Vallespín Muniesa Angélica Fernández-Morales 


\section{Organization}

\section{Honor Committee}

Gioseppe Amoruso

Massimiliano Campi

Enrique Solana

Carlo Bianchini

Pilar Chías

Ángela García

Cesare Cundari

Mario Docci

Francesca Fatta

José Antonio Franco

José María Gentil

Emma Mandelli

Carlos Montes

Pablo Navarro

Rossella Salerno

Javier Seguí
Politecnico di Milano, Italy

Università degli Studi di Napoli Federico II, Italy

Universidad de Las Palmas, Spain

Sapienza Università di Roma, Italy

Universidad de Alcalá, Spain

Universitat Politècnica de València, Spain

Sapienza Università di Roma, Italy

Sapienza Università di Roma, Italy

Università degli Studi di Reggio Calabria, Italy

Universidade da Coruña, Spain

Universidad de Sevilla, Spain

Università degli Studi di Firenze, Italy

Universidad de Valladolid, Spain

Universitat Politècnica de València, Spain

Politecnico di Milano, Italy

Universidad Politécnica de Madrid, Spain

\section{International Scientific Committee}

\author{
Luis Agustín \\ Salvatore Barba \\ Stefano Bertocci \\ Ignacio Cabodevilla \\ José Calvo \\ Mara Capone \\ Eduardo Carazo \\ Noelia Cervero
}

\author{
Universidad de Zaragoza, Spain \\ Università degli Studi di Salerno, Italy \\ Università degli Studi di Firenze, Italy \\ Universidad de Zaragoza, Spain \\ Universidad Politécnica de Cartagena, Spain \\ Università degli Studi di Napoli Federico II, Italy \\ Universidad de Valladolid, Spain \\ Universidad de Zaragoza, Spain
}


Cesare Verdoscia

Emanuela Chiavoni

Javier Domingo

Ernesto Echeverría

Angélica Fernández

José Javier Gallardo

Antonio García-Bueno

Aitor Goitia

Elsa Gutiérrez

Luis Hermida

Iñigo León

Mercedes Linares

Jorge Llopis

Carlos L. Marcos

Barbara Messina

Antonio Millán

Marta Quintilla

Javier Raposo

Ernest Redondo

Simona Salvo

Roberta Spallone

Manuel Ródenas

Karen Sanabria

Miguel Sancho

Aurelio Vallespín
Politecnico di Bari, Italy

Sapienza Università di Roma, Italy

Universidad de Zaragoza, Spain

Universidad de Alcalá, Spain

Universidad de Zaragoza, Spain

Universidad de Zaragoza, Spain

Universidad de Granada, Spain

Universidad CEU San Pablo de Madrid, Spain

Universidad de Las Palmas, Spain

Universidade da Coruña, Spain

Universidad del País Vasco, Spain

Universidad de Sevilla, Spain

Universitat Politècnica de València, Spain

Universidad de Alicante, Spain

Università degli Studi di Salerno, Italy

Universitat Politècnica de Catalunya, Spain

Universidad de Zaragoza, Spain

Universidad Politécnica de Madrid, Spain

Universitat Politècnica de Catalunya, Spain

Sapienza Università di Roma, Italy

Politecnico di Torino, Italy

Universidad Politécnica de Cartagena, Spain

Universidad Tecnológica de La Habana José

Antonio Echeverría, Cuba

Universidad de Zaragoza, Spain

Universidad de Zaragoza, Spain

\section{Peer Reviewers Committee}

\author{
Luis Agustín \\ Ángel Allepuz \\ Antonio Álvaro \\ Antonio Amado \\ Antonio Luis Ampliato \\ Federico Arévalo \\ Salvatore Barba \\ Stefano Bertocci \\ Daniela Besana \\ Antonio Bixio \\ Alberto Bravo \\ Ignacio Cabodevilla \\ Marianna Calia
}

Universidad de Zaragoza, Spain

Universidad de Alicante, Spain

Universidad de Valladolid, Spain

Universidade da Coruña, Spain

Universidad de Sevilla, Spain

Universidad de Sevilla, Spain

Università degli Studi di Salerno, Italy

Università degli Studi di Firenze, Italy

Università di Pavia, Italy

Università degli Studi della Basilicata, Italy

Universidad de Las Palmas, Spain

Universidad de Zaragoza, Spain

Università degli Studi della Basilicata, Italy 
José Calvo

Massimiliano Campi

Mara Capone

Eduardo Carazo

Noelia Cervero

Mauro Chiarella

Stefano Chiarenza

Emanuela Chiavoni

Giuseppe D'acunto

Pierpaolo D'agostino

Giuseppe Damone

Francesco Di Paola

Jorge Domingo

Ernesto Echeverría

Angélica Fernandez

José Ángel Fernández

Juan José Fernández

Fausta Fiorillo

Riccardo Florio

Noelia Galván

Antonio Gámiz

Rodrigo Garcia

Antonio García-Bueno

Francisco García

Josefina García

Javier García-Gutiérrez

Jorge García-Valldecabres

María Teresa Gil

Manuel Giménez

Andrea Giordano

Aitor Goitia

Roberto Goycoolea

Francisco Granero-Martin

Alessandro Greco

Alberto Grijalba

Elsa M. Gutiérrez

Luis Hermida

Francisco Hidalgo

Ricardo Irles

Sonia Izquierdo

Pedro Miguel Jiménez

Pablo J. Juan

Emanuela Lanzara

Iñigo León
Universidad Politécnica de Cartagena, Spain

Università degli Studi di Napoli Federico II, Italy

Università degli Studi di Napoli Federico II, Italy

Universidad de Valladolid, Spain

Universidad de Zaragoza, Spain

Universidad Nacional del Litoral/CONICET, Argentina

Università San Raffaele Roma, Italy

Sapienza Università di Roma, Italy

Università IUAV di Venezia, Italy

Università degli Studi di Napoli Federico II, Italy

Università degli Studi della Basilicata, Italy

Università degli Studi di Palermo, Italy

Universidad de Alicante, Spain

Universidad de Alcalá, Spain

Universidad de Zaragoza, Spain

Universidade da Coruña, Spain

Universidad de Valladolid, Spain

Politecnico di Milano, Italy

Università degli Studi di Napoli Federico II, Italy

Universidad de Valladolid, Spain

Universidad de Sevilla, Spain

Universidad del Bío-Bío, Chile

Universidad de Granada, Spain

Universidad de Alicante, Spain

Universidad Politécnica de Cartagena, Spain

Universidad Politécnica de Madrid, Spain

Universitat Politècnica de València, Spain

Universitat Politècnica de València, Spain

Universitat Politècnica de València, Spain

Università degli Studi di Padova, Italy

Universidad CEU San Pablo de Madrid, Spain

Universidad de Alcalá, Spain

Universidad de Sevilla, Spain

Università di Pavia, Spain

Universidad de Valladolid, Spain

Universidad de Las Palmas, Spain

Universidade da Coruña, Spain

Universitat Politècnica de València, Spain

Universidad de Alicante, Spain

Universidad CEU San Pablo de Madrid, Spain

Universidad Politécnica de Cartagena, Spain

Universidad de Alicante, Spain

Università degli Studi di Napoli Federico II, Italy

Universidad de País Vasco, Spain 
Massimo Leserri

Marco Limongiello

Mercedes Linares

Placido Lizancos

Jorge Llopis

Massimiliano Lo Turco

Daniel Lòpez

Concepción López

Margarita Lorenzo

Clara Maestre

Elena Teresa Clotilde

Carlos L. Marcos

Ángel Martínez

María Luisa Martínez

Rodolfo Mejías

Ángel Melián

Barbara Messina

Antonio Millán

Cosimo Monteleone

Carlos Montes

María José Muñoz

María Lucía Ojeda

Justo Oliva

Lia M. Papa

Sandro Parrinello

José Javier Pérez

Inés Pernas

Francesca Picchio

José Pinto

Francisco Pinto

Juan Carlos Piquer

Enrique Rabasa

Javier Francisco Raposo

Manuel Alejandro Ródenas

Daniel Rodríguez

Eva Juana Rodríguez

Pablo Rodríguez-Navarro

Andrea Rolando

Gabriele Rossi

Adriana Rossi

Michele Russo

Maialen Sagarna

Karen Sanabria
Universidad Pontificia Bolivariana, Colombia

Università degli Studi di Salerno, Italy

Universidad de Sevilla, Spain

Universidade da Coruña, Spain

Universitat Politècnica de València, Spain

Politecnico di Torino, Italy

Universidad de Valladolid, Spain

Universitat Politècnica de València, Spain

Universidade da Coruña, Spain

Universidad CEU San Pablo de Madrid, Spain

Politecnico di Torino, Italy

Universidad de Alicante, Spain

Universidad Politécnica de Madrid, Spain

Universidad de Las Palmas, Spain

Universidad de Costa Rica

Universidad de Las Palmas, Spain

Università degli Studi di Salerno, Italy

Universitat Politècnica de Catalunya, Spain

Università degli Studi di Padova, Italy

Universidad de Valladolid, Spain

Universidad Politécnica de Madrid, Spain

Universidad de Las Palmas, Spain

Universidad de Alicante, Spain

Università degli Studi di Napoli Federico II, Italy

Università degli Studi di Pavia, Italy

Universidad del País Vasco, Spain

Universidade da Coruña, Spain

Università degli Studi di Pavia, Italy

Universidade de Lisboa, Portugal

Universidad de Sevilla, Spain

Universitat Politècnica de València, Spain

Universidad Politécnica de Madrid, Spain

Universidad Politécnica de Madrid, Spain

Universidad Politécnica de Cartagena, Spain

Universidad de Guadalajara, México

Universidad CEU San Pablo de Madrid, Spain

Universitat Politècnica de València, Spain

Politecnico di Milano, Italy

Politecnico di Bari, Italy

Università degli Studi della Campania Luigi

Vanvitelli, Italy

Sapienza Università di Roma, Italy

Universidad del País Vasco, Spain

Universidad Tecnológica de la Habana José

Antonio Echeverría, Cuba 
Miguel Sancho

Alberto Sdegno

Marina Sender

María Senderos

Juan Serra

Ana Torres

Aurelio Vallespín
Universidad de Zaragoza, Spain

Università degli Studi di Udine, Italy

Universitat Politècnica de València, Spain

Universidad del País Vasco, Spain

Universitat Politècnica de València, Spain

Universitat Politècnica de València, Spain

Universidad de Zaragoza, Spain 


\section{Contents}

Heritage and History

The Chapel of Nuestra Señora de los Ojos Grandes . . . . . . . . . . 3

Luis Hermida González

Virtual Musealization Using Video Game Engines.

The Museu à beira do oceano by Lina Bo Bardi

Adrián Sempere-Valenciano, Daniel V. Martín-Fuentes, and Pedro M. Cabezos-Bernal

Constantine in Madrid: The Honorary Arches by Antonio López

Aguado and Custodio Moreno in 1816 and $1829 \ldots \ldots \ldots 25$

Aitor Goitia Cruz

Architectural (and Film) Heritage in Classic Hollywood Movies . . . . . 34

Pedro Molina-Siles and Hugo Barros Costa

Drawing: The Mirror Where the Absent is Looked. The Case

of Monteria Public Market Designed by Horacio Rodriguez . . . . . . . . 45

Massimo Leserri, Alma Benitez, and Dayan Guzmán

Graphic Heritage: 15 Graphic Works Explain Private

Architecture Drawings of the City of Alicante During

the Academic Cycle (1752-1860). A.M.A. Archives

Jorge Domingo-Gresa

The 3D Digital Matrix and the Minaret Tower of Sta.

María in Tauste

Enrique Sancho Pereg, Francisco González Quintial,

and Jaime Carbonel Monguilan

The Shearing Building, an Industrial Typology Developed During

the XVII Century. Features and Details

Nicolás Gutiérrez Pérez 
Graphic Study of the Former Headquarters of the Heart of Mary School of Zamora: An Educational Complex that Died of Success

Daniel López Bragado and Víctor-Antonio Lafuente Sánchez

Surveying in Architectural Intervention of the Collapse of the International Style. The Case of the Puerta de la Carne Market

Federico Arévalo-Rodríguez and Eduardo Martínez-Moya

A Graphic Description of the Religious Heritage of the Medina of Asilah: Mosques, Zaouias and Shrines

Jaime Vergara-Muñoz and Miguel Martínez-Monedero

Detecting and Documenting Hidden Heritage with GPR Techniques . . .

Juan Saumell Lladó, Luis Mariano del Río Pérez,

José Carlos Salcedo Hernández, and Javier Mirabel

Understanding the Roman Baths at Fiesole: From 3D

Documentation to Geometrical and Functional Analysis

Alessandro Nocentini and P. Gregory Warden

Analysis and Proposal for the Chromatic Recovery of the Façade

of the Church of Our Lady of the Angels, in Tuéjar

Irene de la Torre Fornés, Juan Serra Lluch, and Ana Torres Barchino

The Casa de los Pinelo in Seville According to a Text from 1542 . . . . 163

Pedro Barrero-Ortega and Antonio Gámiz-Gordo

Definition of a Protocol for Information Management

and the Creation of the HBIM Model

Jorge García-Valldecabres, Concepción López González, and Luis Cortes Meseguer

Design and Construction of the Plaster Ceilings of Gaudí's La

Pedrera, Unpublished Notes from a 1909 Sketchbook

Galdric Santana Roma

Parallelisms Between the Almo Collegio Borromeo of Pavia and the Colegio Real de Corpus Christi of Valencia . . . . . . . . . . . . . . 202 Concepción López González, Daniela Besana, and Jorge García-Valldecabres

The Unpublished Drawings of the San Acasio Building (Seville) of the Architect Marrón y Ranero

Mercedes Linares Gómez-del-Pulgar and Antonio Tejedor-Cabrera

The Decommissioned Petrol Station in Piazza Manno

in Oristano (Italy)

Vincenzo Bagnolo and Marcello Rosas 
Traces of the Octagonal Crossing of the Cathedral of Pavia

Luis Cortes Meseguer, Daniela Besana, and Concepción López González

Layout, Construction and Surveying of Curvilinear Ribs in Late

Gothic Vaults. The Case of Priego

Ana López-Mozo, Enrique Rabasa-Díaz, and Miguel Ángel Alonso-Rodríguez

Art Nouveau Schools in the Gothic Cloister of San Francisco de Betanzos

Antonio Amado Lorenzo, Marta Colón Alonso, and Fernando Fraga López

Restoration of the Oil Factory of Marxalenes Park 268

Marina Sender Contell and Manuel Giménez Ribera

Analysis and Geometric Modelling of Stellar Vaults

of the Mediterranean Gothic in HBIM Environment

Vincenzo Bagnolo and Raffaele Argiolas

Fusion of VR and AR Strategies for Immersion in Lost

Historical-Architectural Heritage . . . . . . . . . . . . . . . . . . . . . . . . . . . . 289

Mauro Costa Couceiro and Rui Lobo

The Project of Tiburzio Spannocchi for the Fortification

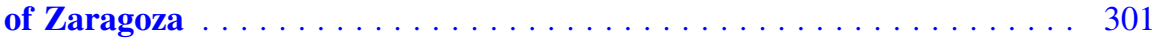

Ignacio Cabodevilla-Artieda

The BIM Model for Existing Building Heritage:

From the Geometric Data Acquisition

to the Information Management .

Carlo Giannattasio, Lia Maria Papa, Pierpaolo D’Agostino,

and Saverio D'Auria

Graphical Restoration of the Cupola of the Tower

of San Bartolomé de Sevilla Based on Historical Photographs .

María Dolores Robador González, Antonio J. Albardonedo Freire, and Domenico Debenedictis

Architecture by Italian Designers and Builders in Buenos Aires.

Knowledge and Valorisations of the Heritage . . . . . . . . . . . . . 333

Maria Pompeiana Iarossi, Giampiero Mele, Francesca Di Geronimo, and Cecilia Santacroce

Visual History of the Sacred Mounts System on Alps.

Representations, Iconologies, Symbols

Anna Marotta 
The Catalan Farmhouse: Evolution and Adaption 367

Carmen Escoda Pastor, Jordi Xiqués Triquell, and Jeongsoo Park

Documentation, Conservation and Reconstruction of the Heritage

of the Middle Kingdom of Thebes, Egypt .

Ernesto Echeverría Valiente, Fernando da Casa Martín, and Flavio Celis D'Amico

Conservation of Contemporary Heritage, Cylindrical

Concrete Sheets, Using the New 3D Reconstruction Techniques

Ernesto Echeverría Valiente, Mónica Martínez Martínez, and Gonzalo García-Rosales

The Keystones of the Cross Vaults of the Lonja de Valencia (Spain). Graphical Analysis of the Geometry of the Polar Keystone of the Starry Vault of the Chapel

Esther Capilla Tamborero

The Soul of Mediterranean Gothic: Drawing with Light, Photographing, as a Case Study in the Communication of Architectural Heritage . . . . . . . . . . . . . . . . . . . . . . . . . . . . 414 Javier Fco. Raposo Grau, Ricardo Santonja Jiménez, and Blanca Raposo Sánchez

The Reuse of Cultural Heritage: A Sustainable Strategy Between

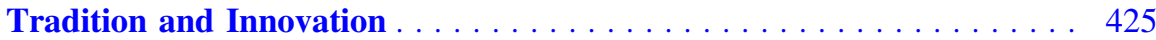

Daniela Besana, Jorge García-Valldecabres, and Luis Cortes Meseguer

Methodological Proposal for the Creation of an Information

System of the Mudejar Architectural Heritage in Aragon

Marta Quintilla Castán

The Grand Staircase in San Xoan de Poio Monastery Inés Pernas Alonso

Protection and Enhancement of Plastic Models:

The Model of the Citadel of Turin

Marco Vitali, Francesca Ronco, and Fabrizio Natta

Graphic Languages and Digital Tools for Communicating and Sharing Heritage

Rossella Salerno

Drawing to Reconstruct. The Convent of Santa Maria della Sanità . 485 Giancarlo Coseglia and Ornella Zerlenga

From the Vascular Art to the Representation of the Scene:

Build, Deconstruct, Rebuild

Andrea Marraffa and Francesca Fatta 
Church of Santa Maria Alemanna in Messina:

Raimondo D'Aronco's Surveys. . . . . . . . . . . . . . . . . . . . . . 513

Adriana Arena

The Participatory Graphics of Cultural Heritage. New Methods

of Analysis, Knowledge and Valorisation

M. Cigola, T. Della Corte, A. Gallozzi, R. Quattrini, L. J. Senatore, and R. M. Strollo

Watchtowers on the Eastern Coast of Andalusia. A Territorial

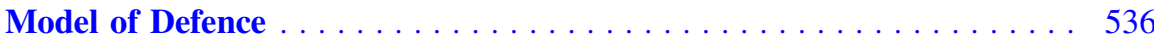

Jose Ramón Delgado Romero

The BIM Methodology: Procedure Improvement vs Innovation

José Javier Gallardo Ortega

Cognitive Systems for the Monumental and Architectural Heritage.

The Aragonese Castle of Taranto .

Cesare Verdoscia, Luis Agustín Hernández, Antonella Musicco, and Riccardo Tavolare

Digital Facsimiles of Architectural Heritage: New Forms of Fruition, Management and Enhancement. The Exemplary

Case of the Ducal Palace at Urbino

Paolo Clini, Ramona Quattrini, Romina Nespeca, Renato Angeloni, and Raissa Mammoli

3D Tools for $\mathrm{CH}$ Documentation

Francesco Di Paola

The Architecture Drawn by Andrea Pozzo. The Church

of S. Thomas of Canterbury in Rome

Marco Fasolo, Matteo Flavio Mancini, and Flavia Camagni

The Historical and Cultural Memory of the Aurelian Walls:

The Archaeological Survey from Ancient Maps

to Contemporary Techniques . . . . . . . . . . . . . . . . . . . 605

Maria Grazia Cianci, Daniele Calisi, and Francesca Paola Mondelli

Diachronic Models of the Façade of Palazzetto Baschenis

Borghese in Rome

Alessandra Marina Giugliano, Maria Martone, and Michele Russo

BIM-Oriented Modelling and Management of Structured

Information for Cultural Heritage

Salvatore Barba, Davide Barbato, Andrea di Filippo,

Raffaella Napoletano, and Federica Ribera 


\title{
Graphic Languages and Digital Tools for Communicating and Sharing Heritage
}

\author{
Rossella Salerno $^{(\bowtie)}$ \\ Politecnico di Milano, Dipartimento di Architettura e Studi Urbani, Milan, Italy \\ rossella.salerno@polimi.it
}

\begin{abstract}
Today languages and digital tools for communicating and sharing heritage seem more and more strictyly connectcted to digital issues.

Much has been done as concerns 3D models embedded in GIS, i.e. employed to document archeological sites and architectural heritage, and more broadly used for increasing tourist attraction places.

Anyway maybe it's possible to go further in achieving a kind of heritage representation ever more addressed to non-specialist persons too, to laypeople.

In such perspective, which goes beyond the boundaries of applied reserach in order to reach social and cultural goals, the mixed use of ICT and web allows to outline new interesting scenarios for heritage, in accordance with EU policies.

The field of heritage representation is therefore still mostly to be investigated, both as regards employing media in portable devices, and as concerns apps for Augmented and Virtual Reality, last but not least for interactive use in georeferred maps.
\end{abstract}

Keywords: Graphic Languages $\cdot$ Digital platforms $\cdot$ Web-based tools

\section{Representing Heritage}

Among manifold interpretative keys suggested by the conference The graphic Heritage. The graphics of Heritage, a possible reading permits to think about two intertwined representation features: on one hand emerges an expertise about tools and procedures which, as experts in drawing, we put into practice in the activities to document heritage; on the other hand, occurs a comparison between a specific legacy of our work and a boost coming from technological and digital innovation.

In the light of what has already been said, the paper aims to focus attention particularly on languages and graphic tools for communicating and sharing heritage, because they seem today more and more strictly connected to digital issues.

Referring to the main approaches recognized by Icomos (International Council on Monuments and Sites) about heritage information, it is well known that two prevailing strategies exist, two approaches different and in the same time related, devoted to study cultural goods, named respectively Presentation and Interpretation (http://icip.icomos. org/ENG/about_missionstatement.html).

The first definition basically refers to information procudures required in analizying a historic or archeological site, elaborated by scholars or professional offices specialised in the field of heritage. 
The second issue, Interpretation, concerns what is meant by the whole of activities, results and creativity led by a site. In such perspective, involving visitors and local people, resident population, is a crucial point.

The Presentation strategy provides a relevant applied topic for those scholars and technicians specialized in digital representation, because it is based on developing 3D models embedded in GIS software, employed i.e. to document archeological sites and historic buildings; it is more broadly used for increasing tourist attraction places. Furthermore, today is easy to produce digital models and render in very short time, so that they can be utilized together to get photorealistic images, able to support visual enjoyment and a better understanding of architectural goods. In this area appear new languages marked by new signs; as wrote Paolo Clini, it deals with: "Panoramic photographic images, holograms, virtual reconstructions, 3D prints [which] multiplied the possibilities of interaction with the end user" [1]; among the last generation devices above mentioned, a specific interest is represented by the photogrammetric technique named Structure from Motion, for digitizing items and allowing their enjoyment, using touch screens and an innovative pseudo-holographic system (Fig. 1).
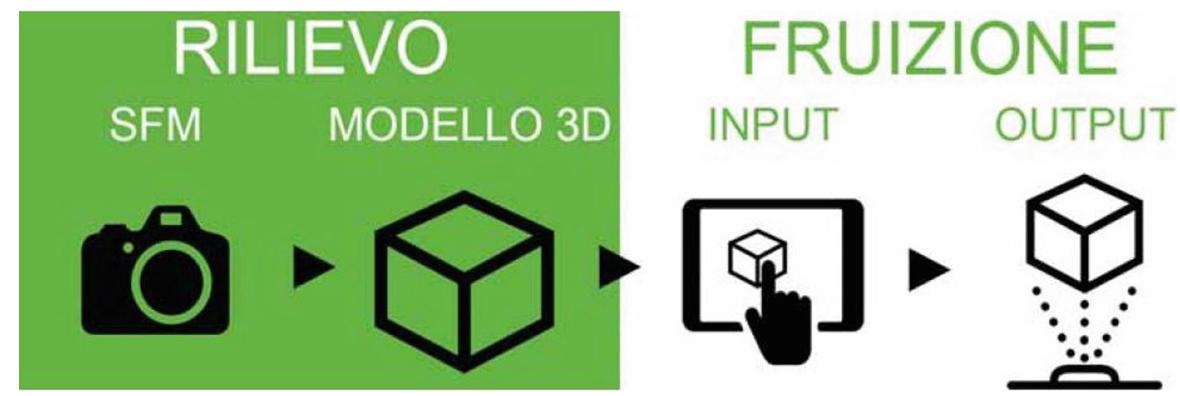

Fig. 1. Workflow of a digitization project: from the photogrammetric to a three-dimensional virtual model, that can be selected using a touchscreen and can be viewed through a holographic system.

A very quick mention has also to be addressed to H-BIM (Heritage Building Information Model), an innovative storage and systematization structure for a whole information about buildings, including geo-referring data in 3D model, and the possibility to visualize at the same time the geometric and the information model.

More, heritage digital models can be focused on communication, as for example it happened in the case of the latest project H2020 INCEPTION (https://www.inceptionproject.eu/en) which, as the website itself shows, has been devoted to realize: "Innovation in $3 D$ modelling of cultural heritage through an inclusive approach for timedynamic $3 D$ reconstruction of artefacts, built and social environments." (Fig. 2, Fig. 3).

However today, after a season of large employment of technology to develop dynamic digital models suitable to share images and information by tablet and smartphone, it seems necessary take a further step toward a kind of heritage 
representation, more and more turned to non specialist persons, to laypeople. Till now, in fact, it appears that the second strategy suggested by Icomos - Presentation - is less practiced; here the key points concern first "communicating" and "sharing" heritage.

\section{(Q) inception}
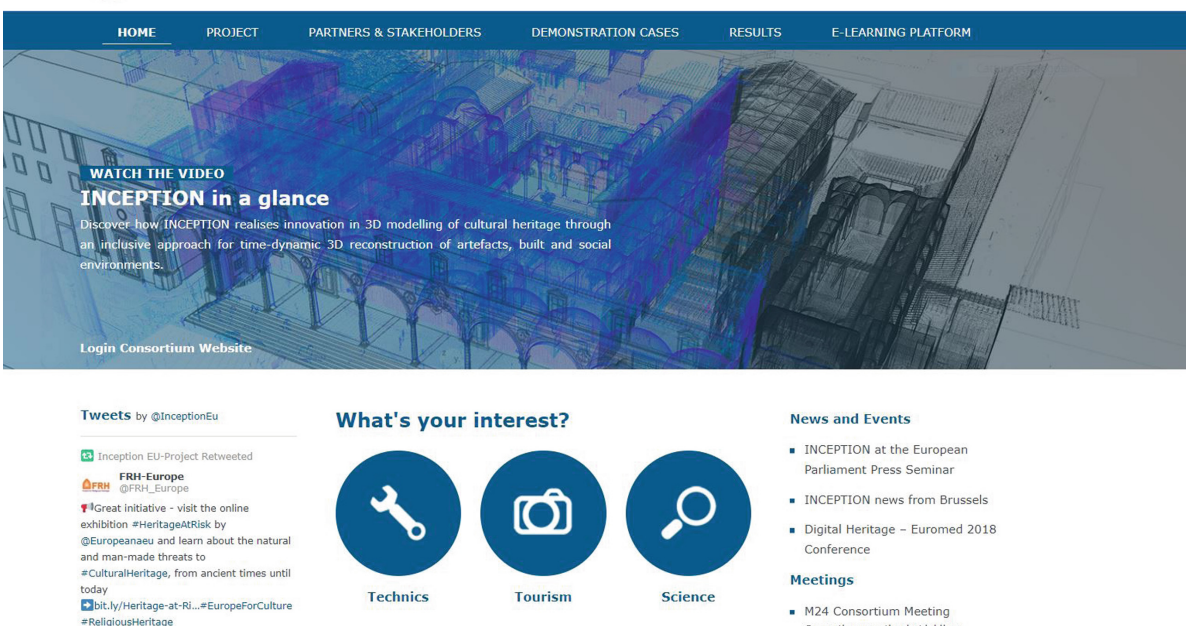

News and Events

- INCEPTION at the European

Parliament Press Seminar

- INCEPTION news from Brussels

- Digital Heritage - Euromed 2018

Meetings

- M24 Consortium Meeting

Consortium meetina in Liubliana,

Fig. 2. Home page from the EU project Inception website: the project is focused on innovation in $3 \mathrm{D}$ modelling for cultural heritage.

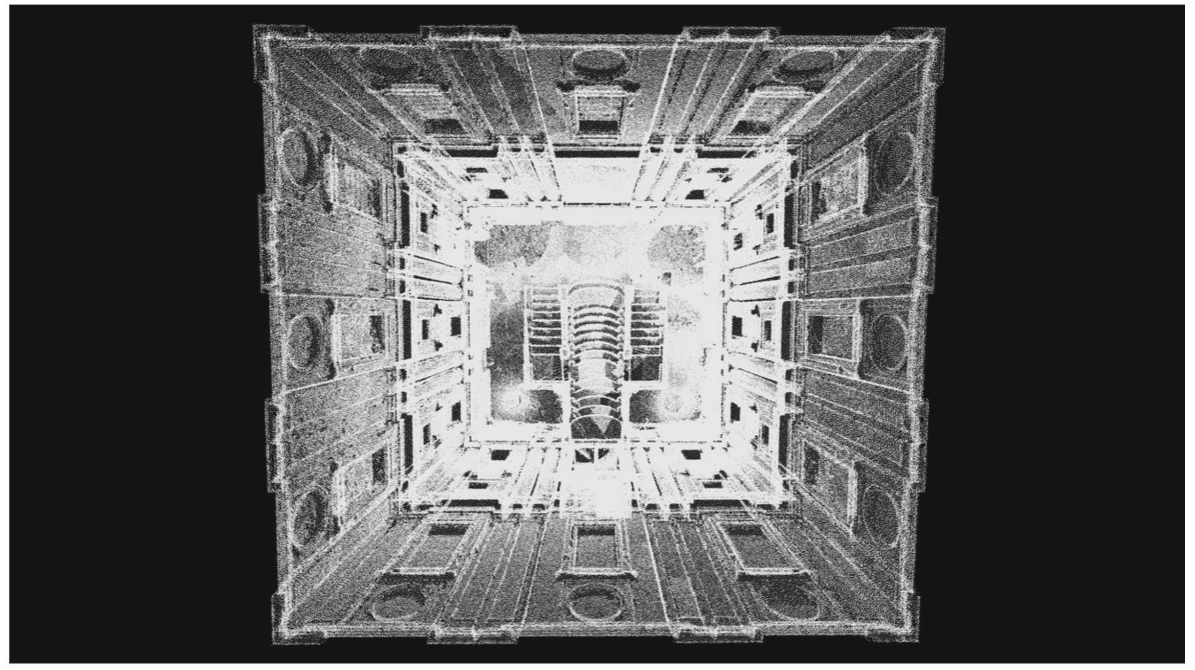

Fig. 3. Digital model of the Biblioteca Laurenziana, Florence, processed in SketchFab, EU project Inception (https://www.inception-project.eu/en/results/3d-models). 
In such perspective, which goes beyond the boundaries of applied research in order to reach social and cultural goals, the mixed use of ICT and web allows to outline new interesting scenarios for heritage, which we can summarize in four main points:

- Data-processing to document places memory;

- Multimedia for cultural heritage;

- Image and information technologies to share places experience;

- Open data and web based tools.

\section{Digitizing Heritage}

So this paper aims to focus on graphic languages and applied technologies' potential, particularly in the cases where heritage approach doesn't follow the usual "top-down" process, rather where it is addressed to promote identification of cultural goods "from below", in other words, when it affects the "bottom-up" process; to be clearer, we mean "top" the procedures employed in the field of heritage by scholars and professionals, and "bottom", the work done by laypeople and living people.

Further the paper is devoted to check how such cutting-hedge procedures have been employed in some experience geared toward EU policy about heritage, a kind of experience which seems featured by an inclusive vision referred to communicate and share cultural heritage.

The European Digital Agenda (ec.europa.eu/digital-agenda/en), on behalf of the last EU Commission, in fact promoted actions for digitizing information and cultural public heritage, supporting administrations, museums, libraries and archives, to complete the computerization of their data, to make them accessible employing Open-Data. It's relevant to remind that Open-Data can be freely used, re-used, re-distributed by everyone, possibly only subject to the need to acknowledge the source, and definitively shared through the same kind of license which they had when initially released.

More recently, EU member states approved a Declaration of Cooperation on Advancing Digitization of Cultural Heritage (2019), a document which defines the basic lines for the next seven years (2020-2027) and highlights the relevance of speeding up technologies application at protecting European cultural heritage.

Three main strategic actions are promoted in such Declaration:

1. A pan-European initiative addressed to $3 \mathrm{D}$ digitizing historic buildings, monuments and sites;

2. Re-use digital resources as well as their innovative use, in order to promote public engagement;

3. Make this cultural field able to generate an impact also in other sectors, through trans-national and cross-sectorial co-operation. 


\subsection{Digital Platforms}

Within such political and cultural framework, a crucial role is assigned to those digital platforms able to create a virtual space for participatory interactions and provide creative contents shared by many people.

The most successful experience till now realized, is represented by Europeana, a digital platform for cultural heritage which makes accessible more than 50 million of records coming from thousands of cultural institutes throughout the European Union (https://www.europeana.eu/portal/en) (Fig. 4). Connecting to Europeana, both citizens and more broadly the cultural and creative field (CCIs), can access European culture through a very wide range of proposals.

Digital content on the platform is made up by images, texts, sound files, video, 3D representations, coming from collections of nearly 3700 libraries, archives, museums, galleries and audio-visual repository over all Europe. Further, digital items can be reused to create educational and learning contents, documentaries, tourism apps, games, entertainments, design tools. Finally, a further aspect that should not be overlooked, Europeana can be considered as a good text to check new technologies and innovative ideas able to improve the users' experience.

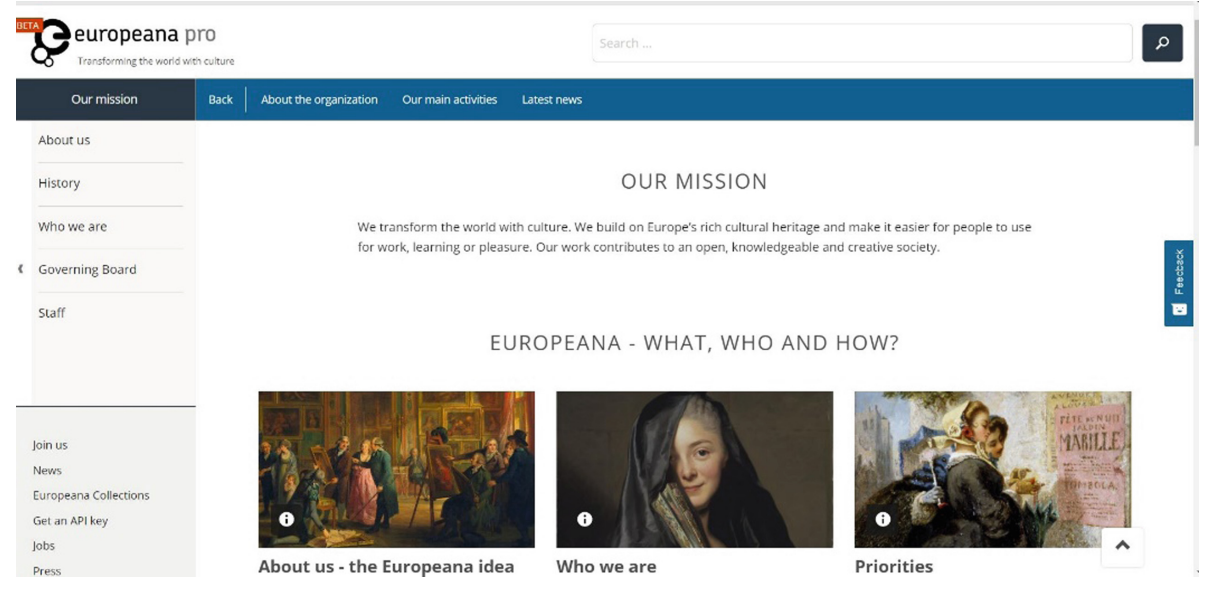

Fig. 4. Europeana Platform. A different way to interpret and approach heritage: Digitizing, Communicating, Sharing.

Among other platforms, it is also useful to remind RICHES, Civic Epistemologies, Photo-consortium and, above all, the latest REACH: RE-designing Access to Cultural Heritage for a wider participation in preservation, (re-)use and management of European culture (https://www.reach-culture.eu/) which, starting with a target devoted to create significative connections between the past and the future, mostly emphasizes all the initiatives suitable to involve the youngest generations, boys school and students 
all over Europe, and gives way to those activities addressed to encourage the engagement by local communities.

The REACH dataset emphasized that dissemination actions intensively apply digital platforms and social media, even if the most challenging trials affect a smart use of digital tools which changed the role played by user/consumer in "active creator" or "active partner", in line with the update models of 3.0 culture [2].

Furthermore, as state Forbes and Colella: "Not all modes of participation in cultural heritage entail the sharing of responsibility and power that defines participatory governance. But they all bear witness to the increasing interest, especially in the twentyfirst century, in democratizing access to culture, and opening up the fruition, management and preservation of heritage to ensure the active and effective collaboration of communities, neighborhoods and individuals." [3].

As concerns actions inspired to "bottom-up" approaches and drawn on digital technologies resources, useful to get a vast increase in participation to produce contents, it is very interesting to underline the employ of community mapping; in that case, the value of participatory approach consists in creating new opportunities, so to elaborate and share legacy forms considered collectively relevant.

Technologies play a strategic role also once citizens are invited to become storytellers; among the very numerous platforms, the Pluggy Project tests the co-operative practices devoted to "share curation" of contents regarding inheritance, so aiming to emphasize a common expertise rather than a formal artistic education: in that case, users are allowed to create virtual exhibitions, hosted rightly on the social platform itself.

\subsection{Sharing Heritage Digitally}

The European Year of Cultural Heritage 2018 provided a large space for the European Framework for Action on Cultural Heritage which, in a meaningful way, took the form around the topic Sharing Heritage: "The European Year of Cultural Heritage - so it is possible to read in the introductory text on the website - aims to make cultural heritage accessible to all by removing social, cultural and physical barriers. The year also aims to engage local communities and help people to rediscover how much of our heritage is shared. Putting our common values, shared history and heritage in the spotlight can contribute to and reinforce a sense of belonging in Europe, and ultimately bring us closer together."

Further, a special space has been dedicated to the issue "Sharing heritage digitally"; within this frame, have been promoted the following three projects for inviting citizens to share legacy through digital tools:

1. Story Map, an interactive interface for cultural heritage, designed on a cartographic base and suitable for help users to explore their nearest initiatives within the framework of the European Capitals of Culture, as well within the sites marked by the European Heritage Label (Fig. 6);

2. Europeana Migration and Europeana 1914-1918 Collections, a whole of stories collections shared by images, each one dedicated to the topics of the migration and of the Great War; 
3. \#Ode2Joy Challenge, promoted by Europa Nostra, addressed to create video on the issue, suitable to be posted subsequently on social media.

These measures prove a different way to understand, communicate and share heritage; here graphic languages, connected to user-friendly digital tools, can lead to a deeper awareness and provide a wider knowledge.

In that respect, the heritage representation framework can provide further effective instruments, both as concerns the employment of media on portable devices, and for the Augmented and Virtual Reality apps, and finally for the manifold interactive ways based on georeferred maps (Fig. 5).

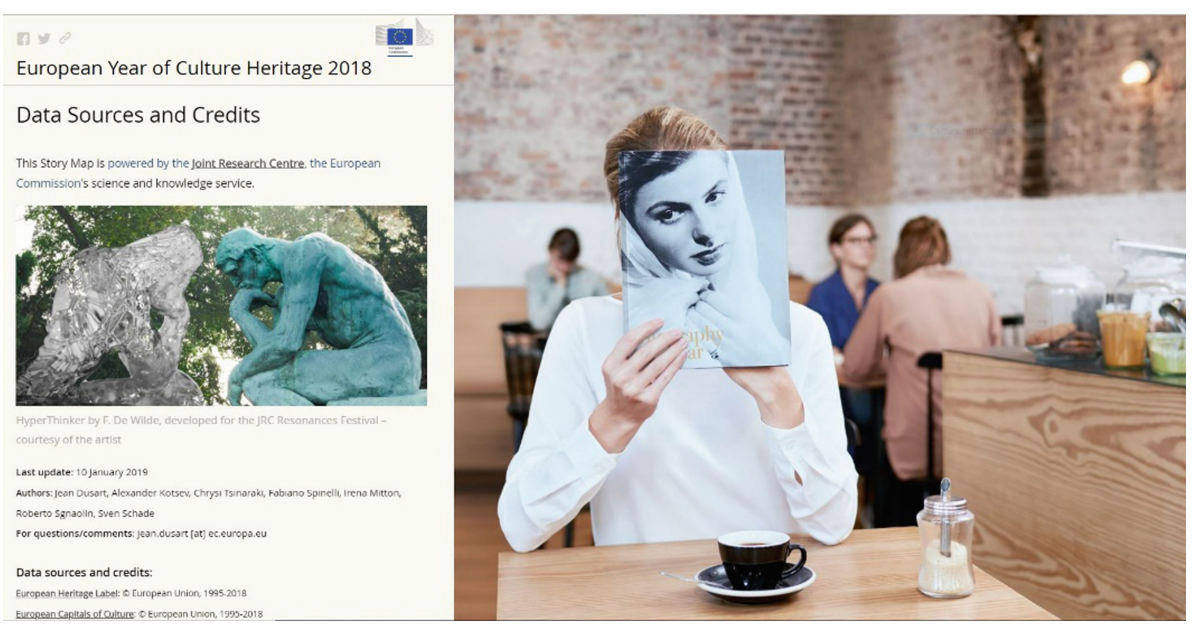

Fig. 5. European Year of Culture Heritage 2018, Story Map, Creative images and media from the interactive interface for cultural heritage map-based. (http://eucommission.maps.arcgis.com/ apps/MapJournal/index.html?appid=e3e538d4e4b743c8a6bc7a363fbc2310)

\section{A Case in Point}

Considering as benchmark the events framework realized in the context of the European Year of Cultural Heritage 2018, we easily understand that numbers are meaningful: 11.700 events in 37 countries, for a total of 6.260 .000 participants. The activities organized over this heritage year cannot be considered exhaustive as regards sharing practices of legacy, anyway as a whole, they are able to help to better comprehend the topic, both for its size and for the interest raised.

Taking into account such background, we propose to analyze an interesting case study from the point of view of the diversified range of technologies employed in sharing information and knowledge about local heritage: this is the "ScaR Project" 
(School Activates Resources), a research project supported by the Politecnico di Milano, which focuses on school and culture as main drivers in enhancing territory and so providing social cohesion (www.scar.polimi.it).

The project goal is overall devoted to raise young's people awareness of their common cultural heritage, considering such action crucial in order to promote an intergenerational and intercultural dialogue in fragile contexts like urban peripheries; the project strategy has been centered in activities to involve students, assigning them a new role, so trying to change their position from cultural heritage users to principal subjects responsible in knowing and promoting them.

As we previously said, in order to reach the target, the ScaR project utilizes a wide range of activities employing technologies: digital storytelling, multimedia guides for tourists, virtual and augmented reality focused on communicating cultural heritage, urban design simulation, urban gaming...

The students, considered like actors in a discovering process, in practices of knowing, interpreting, representing, communicating their local heritage, till now not vey known or unfamiliar for the actors themselves, have been brought to discover, understand, re-formulate, communicate local legacies and so doing, "making them own", realizing a process that helps them to be more conscious citizens for the future.

We now attempt to shortly outline which are the main technologies applied and how they have been used:

1. Digital Storytelling: these digital "story" steps get started defining subjects and topics for the story itself and then move to frame the storyboard, that is the set of chapters narration; what follows it is setting up the students by groups, which has the task of developing the multifaceted aspects in the story; gathering and focusing on information can be done consulting diverse resources, studying the territory, questioning local experts, visiting libraries...; lastly, the process requires a refinement of contents necessary to create the multimedia elements of the story (images, drawings, audio, music, video...).

2. The Digital Atlas of Memories: it is a practice aiming to bring together students and teachers in a participatory action addressed to cultural heritage mapping. As the authors wrote: "A web platform developed to support participatory digital mapping processes is used for this activity: the "Collaborative Gallery", a tool of "Open Deliberative Community Networks" (openDCN) participatory platform, designed to promote User-Generated-Content (UGC) sharing and collaborative content curation [4], creative remix and reuses of cultural content [5], crossmedia integration [6], and the developing of geo-located "media conversation" on places [7]. The Digital Atlas is hosted in the MUMI website, in a section specifically implemented for the SCAR project" [8] (Fig. 6). 


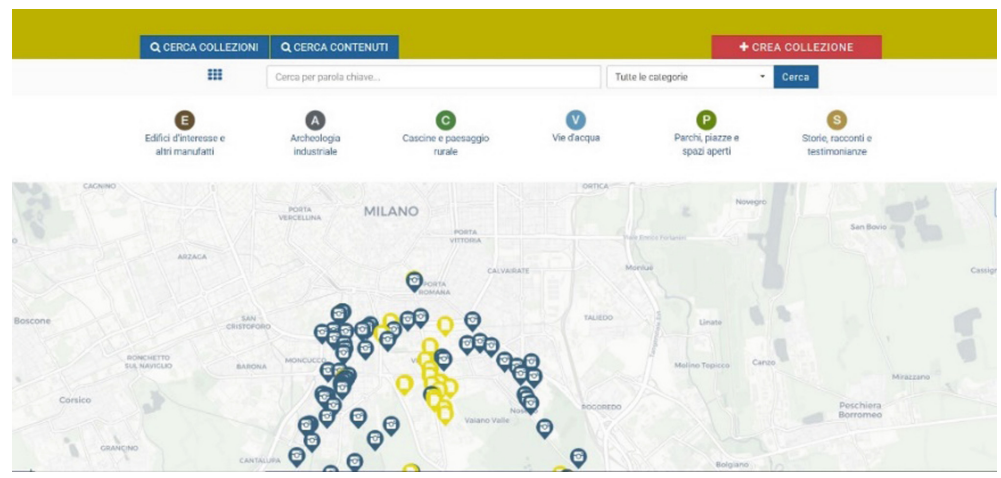

Fig. 6. A screeenshot from The Digital Atlas of memories, MUMI Milano

3. Augmented Reality: virtual and augmented reality are employed in heritage education also through free tools suitable to be used by people non-expert about digital technologies, as for example are software accessible on Google VR (https://vr. google.com); further using $360^{\circ}$ cameras, spherical images and videos in a second step, processed in Google Tour Creator (Fig. 7).

4. Game-Based Learning for Cultural Heritage Education (http://www.gaiasmart. com): in the present case, this is an app allowing people to figure out tangible legacies about cultural heritage through a geogame.

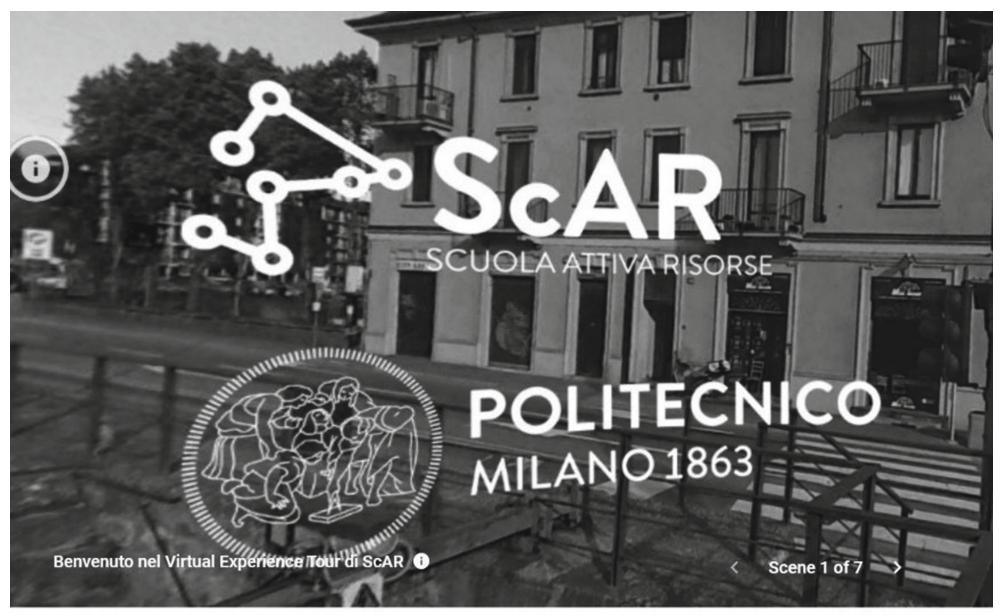

Experience Tour del quartiere Stadera 3 mi piace 181 visualizzazioni

Fig. 7. Frame from the Virtual Tour Experience for the ScaR project. 
Students have been invited to elaborate the game, as a first step focusing on interesting points, and then, implementing contents by images, texts and queries for the game itself.

\section{Conclusion}

A thorough discussion about the whole initiatives carried out over the past European Year of Cultural Heritage 2018 and facing the topic "Sharing Heritage Digitally", could show an overview more structured, as concerns the potential application by digital technologies nowadays available.

Anyway, through the proposed case study, the ScaR project (School Activates Resources), we tried to highlight which are, at moment, application lines more practiced in developing participatory sharing project for cultural heritage.

One last word should be addressed to the feature which seems to underpin the employment of different devices and digital apps, namely a significative shift played by people, not considered only as users but "actors/creators" in their own right.

\section{References}

1. Clini, P., Buroni, P., Ruggeri, L., Angeloni, R.: Pseudo olografia per la fruizione interattiva dei Beni Culturali. Le formelle di Giorgio Martini nel Palazzo Ducale di Urbino/Pseudo holography for interactive fruition of Cultural Heritage. Di Giorgio Martini panels at Ducal Palace of Urbino. In: Salerno, R. (ed.) Proceedings del Convegno Rappresentazione/ Materiale/Immateriale Milano UID 2018, pp. 1059-1066 (2018)

2. Sacco, P.L.: Culture 3.0: a new perspective for the EU 2014-2020 structural funding programme. EENC Paper (2011)

3. Forbes, N., Colella, S.: Embedding engagement: participatory approaches to cultural heritage. Scires-It 9(1), 69-78 (2019)

4. Jenkins, H.: Confronting the Challenges of Participatory Culture: Media Education for the 21st Century. MIT Press, Cambridge (2009)

5. Lessig L.: REMIX - Making Art and Commerce Thrive in the Hybrid Economy. The Penguin Press (2008)

6. Davidson, D.: Cross-media Communications: An Introduction to the Art of Creating Integrated Media Experiences. ETC Press, Pittsburgh (2010)

7. Manovich, L.: The practice of everyday (media) life: from mass consumption to mass cultural production? Crit. Inq. 35(2), 319-331 (2009)

8. Bertone, G., Bordin, M., Casonato, C., Di Blas, N., Pracchi, V., Vedoà, M.: Schools as protagonists in the valorization and communication of their local cultural heritage. In: Proceedings EdMedia + Innovate Learning 2019 - Amsterdam, Netherlands, 24-28 June 2019, pp. 1562-1567 (2019) 